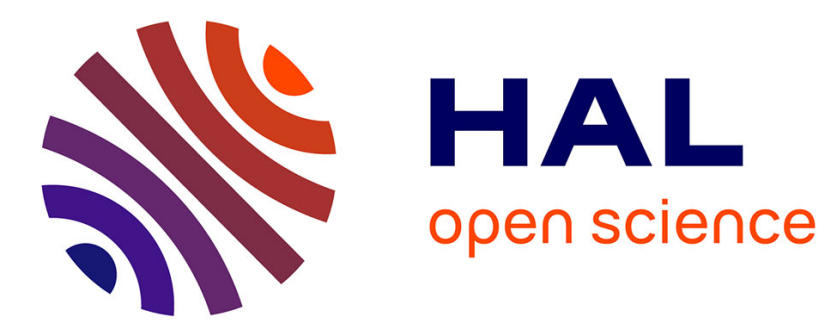

\title{
Layered Electric-Current Approximations of Cylindrical Sources
}

\author{
Andrea Cozza, F Monsef
}

\section{To cite this version:}

Andrea Cozza, F Monsef. Layered Electric-Current Approximations of Cylindrical Sources. Wave Motion, 2016, 64, pp.34-51. 10.1016/j.wavemoti.2016.03.002 . hal-01286537

\section{HAL Id: hal-01286537}

https://hal-centralesupelec.archives-ouvertes.fr/hal-01286537

Submitted on 11 Mar 2016

HAL is a multi-disciplinary open access archive for the deposit and dissemination of scientific research documents, whether they are published or not. The documents may come from teaching and research institutions in France or abroad, or from public or private research centers.
L'archive ouverte pluridisciplinaire HAL, est destinée au dépôt et à la diffusion de documents scientifiques de niveau recherche, publiés ou non, émanant des établissements d'enseignement et de recherche français ou étrangers, des laboratoires publics ou privés. 


\title{
Layered Electric-Current Approximations of Cylindrical Sources
}

\author{
A. Cozza $^{\mathrm{a}}, \mathrm{F} \cdot$ Monsef $^{\mathrm{a}}$ \\ ${ }^{a}$ Physique et Ingénierie de l'Électromagnétisme \\ Group of Electrical Engineering of Paris (GeePs), CNRS UMR 8507, CentraleSupelec - Univ Paris-Sud - UPMC \\ 11 rue Joliot-Curie, Plateau de Moulon, 91192 Gif-sur-Yvette, France. \\ Contact : andrea.cozza@centralesupelec.fr
}

\begin{abstract}
Formulations of the equivalence theorem only involving electric currents either imply non-null inner fields, or the inclusion of a bulk perfect magnetic conductor. The possibility of maintaining a homogeneous background medium across the equivalent-current surface and null inner fields without recurring to magnetic currents is here investigated for the case of cylindrical sources. The proposed procedure keeps using the definition of equivalent electric currents as found in A. Love's theorem, introducing further layers of auxiliary electric currents to mimic the role of the missing magnetic ones. Optimal scalar coefficients applied as weights on each layer current are found, so to minimize the mean quadratic error in the fields radiated outside the auxiliary shell region, while at the same time minimizing internal radiation. The procedure is inherently well-conditioned, thanks to the reduction in the number of degrees of freedom involved, equal to the number of layers, as forced by a regression approach. A thorough numerical analysis is presented, where residual errors are found to be in the range of a few percent points.
\end{abstract}

Keywords: Cylindrical sources, equivalent currents, modal theory, antennas.

\section{Introduction}

Equivalence theorems are a workhorse of antenna theory, widely applied in order to simplify the analysis and the computation of fields generated by a source of radiation. Initially derived by Love and MacDonald more than a century ago [1, 2], they can be flexibly declined into versions that can suite a large number of practical configurations [3, 4, 5]. Independently from the specific version, they all share the same basic idea, illustrated in Fig. 1: given an original source $\Gamma$, identify a spatial distribution of equivalent currents over a closed surface $\Sigma$ containing $\Gamma$, such that these currents generate a perfect reproduction of the field distribution expected for $\Gamma$ over $\Omega_{e}$, outside $\Sigma$. Analogue versions exist for a source $\Gamma$ outside $\Sigma$, a case not treated in this paper.

Referring to the nowadays standard formulation due to Schelkunoff [6], equivalent electric, $\boldsymbol{J}_{\text {eq }}(\boldsymbol{r})$, and magnetic, $\boldsymbol{M}_{\text {eq }}(\boldsymbol{r})$, currents are required, if the equivalent problem in Fig. 1(b) is to work in a homogeneous medium across the surface $\Sigma$. In this case, it can be shown that [1]

$$
\begin{aligned}
\boldsymbol{J}_{\mathrm{eq}}(\boldsymbol{r}) & =\delta\left(\boldsymbol{r}-\boldsymbol{r}_{\Sigma}\right) \hat{\boldsymbol{n}}\left(\boldsymbol{r}_{\Sigma}\right) \times \boldsymbol{H}(\boldsymbol{r}) \\
\boldsymbol{M}_{\mathrm{eq}}(\boldsymbol{r}) & =\delta\left(\boldsymbol{r}-\boldsymbol{r}_{\Sigma}\right) \boldsymbol{E}(\boldsymbol{r}) \times \hat{\boldsymbol{n}}\left(\boldsymbol{r}_{\Sigma}\right),
\end{aligned}
$$

where $\boldsymbol{E}(\boldsymbol{r})$ and $\boldsymbol{H}(\boldsymbol{r})$ are, respectively, the electric and magnetic field generated by the original source at the position $\boldsymbol{r}$; $\delta(\cdot)$ is Dirac's delta distribution, here used in order to represent a singular current sheet limited to $\Sigma ; \hat{\boldsymbol{n}}\left(\boldsymbol{r}_{\Sigma}\right)$ is the outward unit vector normal to $\Sigma$, at the position $\boldsymbol{r}_{\Sigma}$. A time convention $\exp (\mathrm{j} \omega t)$ is assumed, but only phasors are shown. All quantities are frequency dependent, but the pulsation $\omega$ is dropped for the sake of brevity.

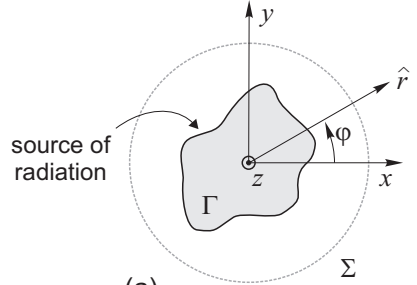

(a)

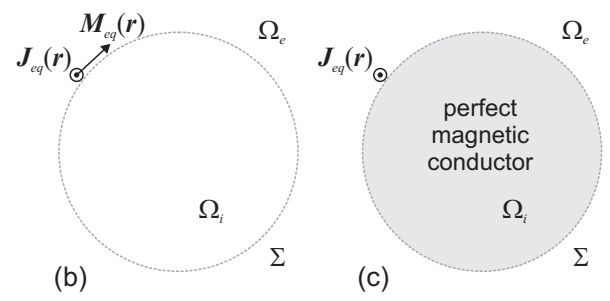

Figure 1: Original source of radiation (a) and two equivalent-current configurations, with a homogeneous background medium using electric and magnetic currents (b) and with only electric currents with the internal region filled by a perfect magnetic conductor (c).

In case the fields radiated by a source were obtained through experiments, both electric and magnetic fields would need to be accessed; in practice, only probes sensitive to one kind of field could be available. Equivalent electric currents suffice if $\Omega_{i}$ is filled with an ideal perfect magnetic conductor (see Fig. 1(c)), as described in [6], ensuring a perfect field reproduction over $\Omega_{e}$. Unfortunately, reproducing this kind of scenario in a numerical model, a bulk material in $\Omega_{i}$ would behave as a scat- 


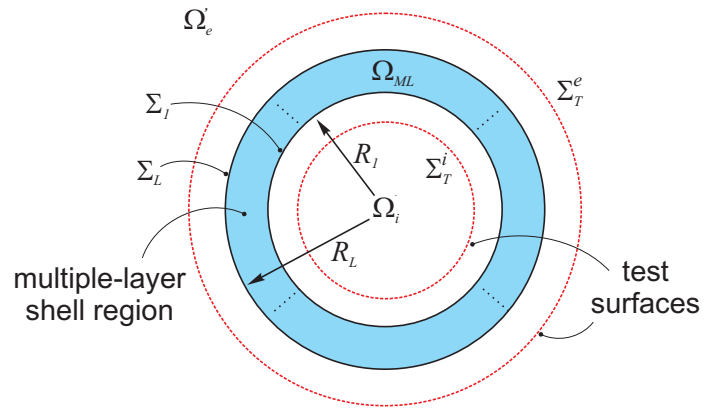

Figure 2: The paradigm considered in this paper: $L$ multiple-layer auxiliary electric-current distributions are found in a shell shown in shade, each occupying a surface $\Sigma_{i}$. Internal and external test surfaces $\Sigma_{T}^{i}$ and $\Sigma_{T}^{e}$, as introduced in sec. 4 , are used for the optimization of the layer weights. The new inner and outer regions are referred to as primed quantities, to differentiate them from those defined in standard equivalent-theorem formulations (see Fig. 1).

terer in case waves impinged onto it. Formulations using only electric currents while keeping a homogeneous medium across $\Sigma$ would be required in this case, as discussed in [7].

Solutions for this problem can be defined, as shown in $[4,8,9,10,11]$, but imply non-null fields in $\Omega_{i}$. This can be a problem since inward radiation inevitably leads to outward waves after propagating through $\Omega_{i}$. In this paper we are interested in equivalent currents that radiate only outside $\Sigma$, as a continuation of the fields radiated by the original source $\Gamma$; therefore, null fields are required in $\Omega_{i}$.

To this end, magnetic equivalent currents could be converted into an additional electric contribution $\boldsymbol{J}_{\mathrm{M}}(\boldsymbol{r})=\nabla \times \boldsymbol{M}_{\text {eq }}(\boldsymbol{r}) / \mathrm{j} \omega \mu$ [12]. But in case of an experimental characterization of the original source, this operation is ill-posed, since it involves estimating derivatives from measurements. Critically, normal derivatives would require sampling fields at different distances from the original source. Moreover, this further electric-current contribution now involves spatial derivatives of a Dirac distribution, which does not correspond to a double-sheet of currents.

In order to overcome these difficulties, this paper suggests using multiple layers of electric currents, defining a shell region $\Omega_{\mathrm{ML}}$ as shown in Fig. 2. The rationale is to make the fields radiated by the different layers of currents interfere, in such a way as to emulate the interplay between fields radiated by electric and magnetic currents in Love's theorem.

Our proposal is not to set up an inverse problem where the equivalent current distributions over each layer would act as unknowns, since this kind of approach is often ill-conditioned, particularly in the case of measured data. We rather attempt extending the use of distributions defined as in (1a) over at least two surfaces, by defining optimal scalar coefficients acting as weights on each of these current distributions. As it will be shown in the rest of the paper, the proposed method, though effective, does not allow a perfect equivalence, hence our referring to these currents as auxiliary rather than equivalent.

\section{Scalar representation of the problem}

In the following we will consider the case of original sources described by means of electric-current distributions confined within a shell region of space $\Omega_{\mathrm{ML}}$ (Fig. 2). For ease of derivation, a cylindrical geometry will be assumed, with translation invariance to axial displacements.

Dealing with cylindrical sources, it is convenient to proceed by separating the original currents modelling the source over the region $\Gamma$ (Fig. 1) into axial and transversal components

$$
\boldsymbol{J}_{S}(\boldsymbol{r})=\mathbf{J}_{\mathrm{S}}^{\|}(\boldsymbol{r})+\mathbf{J}_{\mathrm{S}}^{\perp}(\boldsymbol{r})=\hat{z} J_{\mathrm{S}}^{\| l}(\boldsymbol{r})+\hat{\boldsymbol{\varphi}} J_{\mathrm{S}}^{\perp}(\boldsymbol{r}),
$$

where bold variables represent vector quantities while hatted ones are unit vectors. In order to simplify our derivation, we introduce magnetic currents [12, section 7.12]

$$
\mathbf{M}_{\mathrm{S}}^{\|}(\boldsymbol{r})=\hat{z} M_{\mathrm{S}}^{\|}(\boldsymbol{r})=-\frac{1}{\mathrm{j} \omega \epsilon} \nabla \times \mathbf{J}_{\mathrm{s}}^{\perp}(\boldsymbol{r}) .
$$

In the following, instead of the original current distribution (2) we will consider a source described by axial currents $\mathbf{J}_{\mathrm{S}}^{\|}(\boldsymbol{r})$ and $\mathbf{M}_{\mathrm{S}}^{\prime l}(\boldsymbol{r})$.

Maxwell's equations for cylindrical configurations can be expressed as [12, section 14.1]

$$
\begin{aligned}
\left(\nabla^{2}+\omega^{2} \epsilon \mu\right)^{2} E^{\prime \prime}(\boldsymbol{r}) & =-\mathrm{j} \omega \mu J_{\mathrm{s}}^{\|}(\boldsymbol{r})-\nabla \cdot\left(\hat{z} \times \mathbf{M}_{\mathrm{s}}^{\perp}(\boldsymbol{r})\right) \\
\left(\nabla^{2}+\omega^{2} \epsilon \mu\right)^{2} H^{\prime \prime}(\boldsymbol{r}) & =-\mathrm{j} \omega \epsilon M_{\mathrm{s}}^{\| \prime}(\boldsymbol{r})-\nabla \cdot\left(\hat{z} \times \mathbf{J}_{\mathrm{s}}^{\perp}(\boldsymbol{r})\right),
\end{aligned}
$$

hence describing the source through only axial electric and magnetic currents yields scalar decoupled equations

$$
\begin{aligned}
\left(\nabla^{2}+\omega^{2} \epsilon \mu\right)^{2} E^{\prime \prime}(\boldsymbol{r}) & =-\mathrm{j} \omega \mu J_{\mathrm{s}}^{\|}(\boldsymbol{r}) \\
\left(\nabla^{2}+\omega^{2} \epsilon \mu\right)^{2} H^{\prime \prime}(\boldsymbol{r}) & =-\mathrm{j} \omega \epsilon M_{\mathrm{s}}^{\|}(\boldsymbol{r}),
\end{aligned}
$$

corresponding to a TM (transverse-magnetic) and TE (transverse-electric) problem, respectively. Hence, (6) is solved by

$$
E^{\prime \prime}(\boldsymbol{r})=\frac{\omega \mu}{4} \int_{\Gamma} \mathrm{d} \boldsymbol{r}^{\prime} H_{0}\left(k_{0}\left\|\boldsymbol{r}-\boldsymbol{r}^{\prime}\right\|\right) J_{\mathrm{s}}^{\|}\left(\boldsymbol{r}^{\prime}\right),
$$

where $H_{0}(\cdot)$ is Hankel's function of zero-th order of the first kind (i.e., for an outgoing wave). Equation (8) is also a solution to (7), by substituting electric-field related quantities with magnetic ones, as according to the duality principle; for this reason, the rest of the paper will only focus on the case of axial electric sources. Solutions found for this problem can be readily applied to the case of azimuthal electric sources thanks to (3).

\section{Fields radiated by a single layer of electric currents}

We first seek a modal expansion of the electromagnetic field radiated by the original source in $\Gamma$, by applying the addition theorem to (8), here recalled for the sake of completeness, for the special case of the outgoing Hankel function of the zeroth order

$$
\begin{aligned}
& H_{0}\left(k_{o}\left\|\boldsymbol{r}-\boldsymbol{r}^{\prime}\right\|\right)= \\
& \begin{cases}\sum_{m=-\infty}^{\infty} J_{m}\left(k_{o} r^{\prime}\right) H_{m}\left(k_{o} r\right) \mathrm{e}^{\mathrm{j} m\left(\varphi-\varphi^{\prime}\right)} & r^{\prime}<r \\
\sum_{m=-\infty}^{\infty} H_{m}\left(k_{o} r^{\prime}\right) J_{m}\left(k_{o} r\right) \mathrm{e}^{\mathrm{j} m\left(\varphi-\varphi^{\prime}\right)} & r^{\prime}>r\end{cases}
\end{aligned}
$$


where $J_{m}(\cdot)$ and $H_{m}(\cdot)$ are Bessel's and Hankel's functions of the $m$ th order of the first kind. From (8) and (9) we straightforwardly obtain

$$
E^{\|}(\boldsymbol{r})=\frac{\omega \mu}{4} \sum_{m=-\infty}^{\infty} \gamma_{m} \psi_{m}^{h}(\boldsymbol{r}) \quad \boldsymbol{r} \in \Omega_{e},
$$

having introduced a shorthand representation of cylindrical modes

$$
\begin{aligned}
& \psi_{m}^{h}(\boldsymbol{r})=H_{m}\left(k_{o} r\right) \mathrm{e}^{\mathrm{j} m \varphi} \\
& \psi_{m}^{j}(\boldsymbol{r})=J_{m}\left(k_{o} r\right) \mathrm{e}^{\mathrm{j} m \varphi}
\end{aligned}
$$

and the associated modal weights

$$
\gamma_{m}=\int_{\Gamma} \mathrm{d} \boldsymbol{r}^{\prime}\left[\psi_{m}^{j}\left(\boldsymbol{r}^{\prime}\right)\right]^{*} J_{\mathrm{e}}^{\| \prime}\left(\boldsymbol{r}^{\prime}\right),
$$

with $*$ the complex conjugate.

The magnetic field can also be represented in a similar manner; making use of the electric-field curl equation,

$$
\begin{aligned}
\mathbf{H}^{\perp}(\boldsymbol{r}) & =\frac{\mathbf{j}}{4} \sum_{m=-\infty}^{\infty} \gamma_{m} \nabla \times\left[\hat{\boldsymbol{z}} \psi_{m}^{h}(\boldsymbol{r})\right]= \\
& =\sum_{m=-\infty}^{\infty} \gamma_{m}\left[\hat{\boldsymbol{j}} \frac{\mathrm{j} m}{r}-\hat{\boldsymbol{\varphi}} \partial_{r}\right] \psi_{m}^{h}(\boldsymbol{r}),
\end{aligned}
$$

where $\partial_{r}$ is the first-order derivative taken with respect to the radial distance $r$.

In order to understand what is the effect of removing equivalent magnetic currents in a free-space configuration, we consider a cylindrical surface $\Sigma$, of radius $R$, over which

$$
J_{\mathrm{eq}}^{\| \prime}(\boldsymbol{r})=-\delta(r-R) \sum_{m=-\infty}^{\infty} \gamma_{m} \mathrm{e}^{\mathrm{j} m \varphi} \partial_{r} H_{m}\left(k_{o} r\right)
$$

as given by (1) and (13).

The electric field generated by these currents can be computed by applying (8) to the current distribution (14). The application of the addition theorem (9) yields

$$
\hat{E}^{\prime \prime}(\boldsymbol{r})=\frac{\omega \mu}{4} \sum_{m=-\infty}^{\infty} \gamma_{m} \begin{cases}\eta_{m}^{e}(R) \psi_{m}^{h}(\boldsymbol{r}) & \boldsymbol{r} \in \Omega_{e} \\ \eta_{m}^{i}(R) \psi_{m}^{j}(\boldsymbol{r}) & \boldsymbol{r} \in \Omega_{i}\end{cases}
$$

with

$$
\begin{aligned}
& \eta_{m}^{e}(R)=-\mathrm{j} \pi R J_{m}\left(k_{o} R\right) \partial_{R} H_{m}\left(k_{o} R\right) \\
& \eta_{m}^{i}(R)=-\mathrm{j} \pi R H_{m}\left(k_{o} R\right) \partial_{R} H_{m}\left(k_{o} R\right) .
\end{aligned}
$$

The corresponding magnetic field is

$$
\begin{aligned}
\hat{\mathbf{H}}^{\perp}(\boldsymbol{r})= & \frac{\mathrm{j}}{4} \sum_{m=-\infty}^{\infty} \gamma_{m}\left[\hat{\boldsymbol{r}} \frac{\mathrm{j} m}{r}-\hat{\boldsymbol{\varphi}} \partial_{r}\right] \\
& \begin{cases}\eta_{m}^{e}(R) \psi_{m}^{h}(\boldsymbol{r}) & \boldsymbol{r} \in \Omega_{e} \\
\eta_{m}^{i}(R) \psi_{m}^{j}(\boldsymbol{r}) & \boldsymbol{r} \in \Omega_{i}\end{cases}
\end{aligned}
$$

Fig. 3 shows an example of the electric and magnetic field distributions for the ideal case expected for the equivalence theorem, and those observed in the case where only equivalent
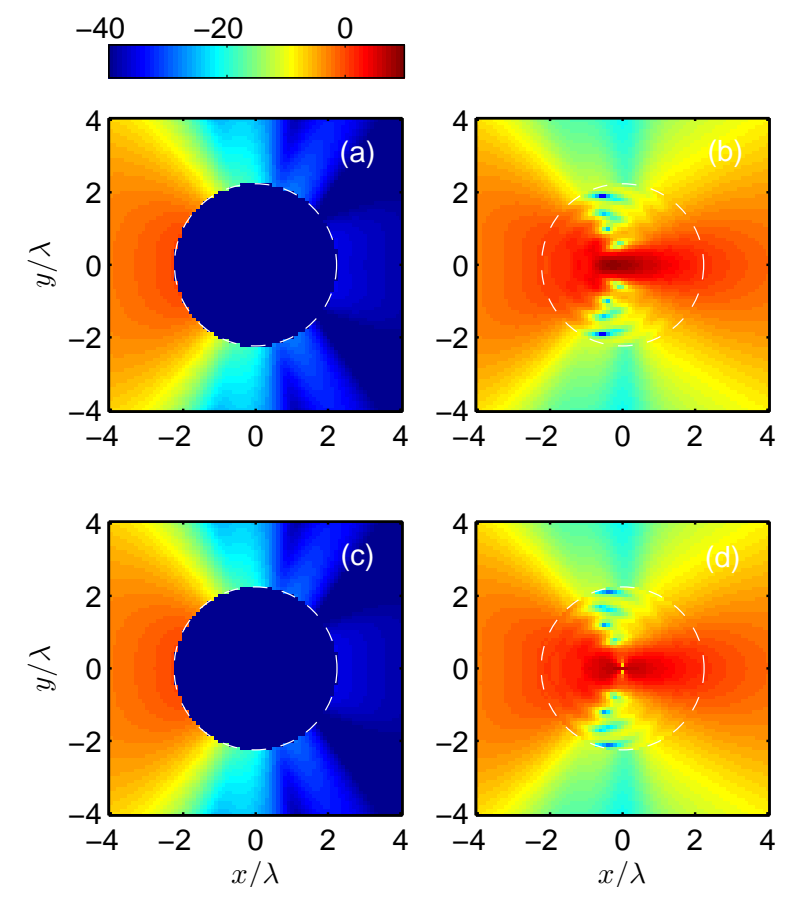

Figure 3: An example of electric (b) and magnetic (d) fields radiated by a single layer of equivalent electric currents, here represented by the dashed circle. Reference field distributions expected for the case of electric and magnetic equivalent currents are shown on the left column, respectively for the electric (a) and magnetic (c) field. Corresponding field distributions generated by the electric currents alone are given on the right column. Data represent the amplitude of the fields measured in $\mathrm{dB}$, normalized to the peak amplitude observed for the reference distribution.

electric currents are present, i.e., a partial application of the equivalence theorem. These results refer to the test-case described in section 8 , dealing with a directive linear source four wavelengths long. This source has a directive leftward radiation.

In the case of partial application of the equivalence theorem, the leftward radiation is well reproduced, whereas a spurious rightward radiation appears. This last is due to the fact that equivalent electric currents that are at the origin of the leftward radiation do also radiate towards the inner region $\Omega_{i}$; this inward radiation would be counterbalanced by equivalent magnetic currents in the equivalence-theorem free-space formulation. Left alone, the inward contribution may focus inside the inner region $\Omega_{i}$ and subsequently diverge rightwards, causing the spurious radiation that did not appear in the original configuration. Hence the need for an alternative mechanism of control of the inward radiation.

\section{Multiple-layer auxiliary currents}

Reproducing the results of Love's formulation would require, apart for a common constant factor, to ensure that $\eta_{m}^{e}(R)=$ $1, \forall m$ and $\eta_{m}^{i}(R)=0, \forall m$. In other words, the $\eta_{m}^{i, e}(R)$ coefficients act as distortions, in the modal expansion of the field distribution generated by the original electric currents $\mathbf{J}_{\mathrm{e}}^{\|}(\boldsymbol{r})$, by 
passing, e.g., for the region external to $\Sigma$, from modal coefficients $\left\{\gamma_{m}\right\}$ to $\left\{\gamma_{m} \eta_{m}^{e}(R)\right\}$.

Our proposal is to introduce multiple layers of auxiliary current distributions, defined as in Fig. 2 over $L$ concentric cylindrical surfaces $\left\{\Sigma_{l}\right\}$, with respective radii $\left\{R_{l}\right\}$. The rational behind this procedure is that by applying different weights to the individual electric current distributions, the resulting overall modal coefficients can be forced to match more closely those expected for (1). The electric currents over each layer is defined as in Love's equivalence theorem, but are further weighted by coefficients $\left\{A_{l}\right\}$, leading to overall fields (15) and (17) with a new set of coefficients

$$
\eta_{m}^{i, e}=\sum_{l=1}^{L} A_{l} \eta_{m}^{i, e}\left(R_{l}\right)
$$

As discussed in the next section, the problem is now to identify the optimal weights $\left\{A_{l}\right\}$ that reproduce as closely as possible the electromagnetic field distribution generated by the original source over $\Omega_{e}^{\prime}$, and null fields over $\Omega_{i}^{\prime}$, while the region of space $\Omega_{\mathrm{ML}}$ occupied by the layers will act as a buffer region of finite thickness.

Residual errors are minimized over test surfaces $\Sigma_{T}^{e}$ and $\Sigma_{T}^{i}$, respectively for external and internal fields. Clearly, the equivalence theorem implies that any choice of these surfaces is equivalent. Therefore, in the following, we assess the accuracy of the approximate field over cylindrical test surfaces $\Sigma_{T}^{e}$ and $\Sigma_{T}^{i}$ of radii $R_{T}^{e}>R_{L}$ and $R_{T}^{i}<R_{1}$, respectively, as illustrated in Fig. 2 . Only errors on tangential fields will be evaluated. Even though the proposed procedure can be extended to any number of layers, we will hereafter set $L=2$, in order to look for solutions offered by the simplest possible case.

The rms errors are here defined as

$$
\begin{aligned}
e_{E}^{2}\left(R_{T}\right) & =(\omega \mu / 4)^{-2} \oint_{\Sigma_{T}} \mathrm{~d} \boldsymbol{r}\left|\hat{E}^{\prime \prime}(\boldsymbol{r})-E^{\prime \prime}(\boldsymbol{r})\right|^{2} \\
e_{H}^{2}\left(R_{T}\right) & =(1 / 4)^{-2} \oint_{\Sigma_{T}} \mathrm{~d} \boldsymbol{r}\left|\hat{\boldsymbol{\varphi}} \cdot\left(\hat{\mathbf{H}}^{\perp}(\boldsymbol{r})-\mathbf{H}^{\perp}(\boldsymbol{r})\right)\right|^{2},
\end{aligned}
$$

perfect reproduction of the original field distributions over $\Omega_{e}^{\prime}$ would now imply the condition $\eta_{m}^{e}=1, \forall m$. Hence, from (10), (13), (15) and (17)

$$
e_{E, H}^{2}\left(R_{T}\right)= \begin{cases}\sum_{m=-\infty}^{\infty}\left|\eta_{m}^{e}-1\right|^{2}\left|\beta_{m}^{E, H}\left(R_{T}\right)\right|^{2} & R_{T}>R_{L} \\ \sum_{m=-\infty}^{\infty}\left|\eta_{m}^{i}\right|^{2}\left|\beta_{m}^{E, H}\left(R_{T}\right)\right|^{2} & R_{T}<R_{1}\end{cases}
$$

where

$$
\beta_{m}^{E}\left(R_{T}\right)= \begin{cases}\gamma_{m} H_{m}\left(k_{o} R_{T}\right) & R_{T}>R_{L} \\ \gamma_{m} J_{m}\left(k_{o} R_{T}\right) & R_{T}<R_{1}\end{cases}
$$

and

$$
\beta_{m}^{H}\left(R_{T}\right)= \begin{cases}\gamma_{m} \partial_{r} H_{m}\left(k_{o} R_{T}\right) & R_{T}>R_{L} \\ \gamma_{m} \partial_{r} J_{m}\left(k_{o} R_{T}\right) & R_{T}<R_{1}\end{cases}
$$

are proportional to the individual modal contributions to the original field distributions required by Love's formulation.

\section{Optimal layer weights}

The errors in (21) are ultimately functions of the layer weights $\left\{A_{l}\right\}$ and should be minimized in order to find an optimal excitation of the auxiliary-current layers. In this respect, all summations will be limited to terms $|m|<M$, where $M$ can be chosen from one of the heuristic criteria routinely applied in electromagnetic theory $[13,14]$.

The simplest option for minimizing the rms errors consists in observing that $e_{E}$ can be interpreted as the length of the difference vector found between two vectors whose scalar elements are respectively $\left\{\beta_{m}^{E} \eta_{m}^{e}\right\}$ and $\left\{\beta_{m}^{E}\right\}$. In this respect, $e_{E}^{2}\left(R_{T}\right), R_{T}>R_{L}$ in (21) can be minimized by means of a leastsquare (LS) approach, by introducing the normal equation [15]

$$
\boldsymbol{\beta}^{E}\left(R_{T}^{e}\right) \boldsymbol{\eta}^{e} \boldsymbol{A}=\boldsymbol{\beta}^{E}\left(R_{T}^{e}\right) \boldsymbol{c}_{1},
$$

where $\boldsymbol{\beta}^{E}=\operatorname{diag}\left\{\beta_{-M}^{E}, \ldots, \beta_{M}^{E}\right\}, \boldsymbol{\eta}^{e}$ is an $2 M+1 \times L$ matrix whose columns are composed by the $\left\{\eta_{m}^{e}\left(R_{l}\right)\right\}, \boldsymbol{A}=$ $\left[A_{1}, \ldots, A_{L}\right]^{T}$ are the $L$ layer weights to be optimized and $\boldsymbol{c}_{a}$ is a $2 M+1$ column vector of elements equal to $a$, here $a=1$. In practice, (24) translates the problem of finding the optimal $\left\{A_{l}\right\}$ such that $\left\{\eta_{m}^{e}\right\} \simeq 1, \forall m \in[-M, M]$, but weighting the individual errors according to the energy contributed by each mode, as assessed by $\left|\beta_{m}^{E}\right|^{2}$.

The LS solution to (24) is thus given by

$$
\boldsymbol{A}=\left[\boldsymbol{\beta}^{E}\left(R_{T}^{e}\right) \boldsymbol{\eta}^{e}\right]^{\dagger} \boldsymbol{\beta}^{E}\left(R_{T}^{e}\right) \boldsymbol{c}_{1}
$$

with $†$ the Penrose-Moore inverse. In general, this solution will depend on the set of LS-weights $\boldsymbol{\beta}$ and therefore on whether one intends to optimize the reproduction of the electric or of the magnetic field. In practice, the solution is weakly dependent on which of the two sets (22) or (23) are chosen, since it can be shown that

$$
\frac{\beta_{m}^{H}(r)}{\beta_{m}^{E}(r)}=\frac{H_{m}^{\prime}\left(k_{o} r\right)}{H_{m}\left(k_{o} r\right)} \simeq \mathrm{j} \quad \forall r \gtrsim W,
$$

with $W$ the maximum transversal dimensions of the source. Hence, the optimization weights $\left\{\beta_{m}^{E, H}\right\}$ are practically proportional one to the other for a given $m$. As a consequence, both electric and magnetic fields will be asymptotically optimized at the same time. This trend is indeed confirmed by the results presented in sec. 8 for external test surfaces, while it is not necessarily the case for inner test surfaces, where choosing between the two sets of $\left\{\beta_{m}\right\}$ can have a measurable effect on the inner error, as shown in sec. 8.4.

\section{Internal \& external optimization}

The above results are divided into internal and external regions defined by the auxiliary-source surfaces. It is therefore natural to wonder if layer weights could be optimized for both regions at the same time, or if any joint optimization can only be obtained by degrading the best performance attainable when dealing only with one region. 
In order to gain some insight into this issue, we recall that for $k_{o} r \gg 1$

$$
H_{m}\left(k_{o} r\right) \simeq \sqrt{\frac{2}{\pi k_{o} r}} \mathrm{e}^{\mathrm{j}\left(k_{o} r-m \pi / 2-\pi / 4\right)},
$$

while $H_{m}^{\prime}\left(k_{o} r\right) \simeq \mathrm{j} H_{m}\left(k_{o} r\right)$. Since $J_{m}\left(k_{o} r\right)=\operatorname{Re} H_{m}\left(k_{o} r\right)$, in case $k_{o} R_{l} \gg 1, \quad \forall l \in[1, L],(16)$ is asymptotically approximated by

$$
\begin{aligned}
& \eta_{m}^{e}(R) \simeq 1-\mathrm{j}(-1)^{m} \mathrm{e}^{2 \mathrm{j} k_{o} R} \\
& \eta_{m}^{i}(R) \simeq-2 \mathrm{j}(-1)^{m} \mathrm{e}^{2 \mathrm{j} k_{o} R}
\end{aligned}
$$

for a single layer of auxiliary electric currents. When applying these results to (18), for $L$ layers,

$$
\begin{aligned}
\eta_{m}^{e} & \simeq \sum_{l=1}^{L} A_{l}-\mathrm{j}(-1)^{m} \sum_{l=1}^{L} A_{l} \mathrm{e}^{2 \mathrm{j} k_{o} R_{l}} \\
\eta_{m}^{i} & \simeq-2 \mathrm{j}(-1)^{m} \sum_{l=1}^{L} A_{l} \mathrm{e}^{2 \mathrm{j} k_{o} R_{l}} .
\end{aligned}
$$

A close look at (28) shows that the $\left\{\eta_{m}^{e}\right\}$ appear to be given by an offset version of the $\left\{\eta_{m}^{i}\right\}$, by a fixed quantity not depending on the modal index $m$. As a result, when the condition of null internal field $\eta_{m}^{i}=0, \quad \forall m$, is fulfilled, $\eta_{m}^{e}=1, \forall m$, hence implying a perfect reproduction of the field topography over the external region. Therefore, internal and external optimization can go hand in hand as the above condition is sufficient to ensure an exact reproduction of the field topographies dictated by Love's formulation of the equivalence theorem, at least within an asymptotic framework. Moreover, (29) also explains how the $L$ layer weights can control $M \gg L$ modal distortions, thanks to the asymptotic factorization of the portions impacted by the $l$ and $m$ indexes.

In practice, the minimization of the metrics in (21) leads to sub-optimal solutions, resulting in inevitably $m$-dependent modal distortions $\left\{\eta_{m}^{i, e}\right\}$. In fact, it should be recalled that the LS problem discussed in sec. 5 is typically overdetermined, since $L \ll M$ in most configurations: in the present paper we will consider $L=2$, while $M \gtrsim 1$ even for weakly directive sources. Under these conditions, the only possibility for acceptable solutions must pass through the minimization of the portion shared by the external and internal distortions, as seen in (29). In any other case, the number of degrees of freedom would be too small with respect to the modal weights to control them independently.

More generally, a joint optimization over the internal and external surfaces can be considered, by extending (24) as

$$
\left[\begin{array}{c}
\boldsymbol{\beta}^{E}\left(R_{T}^{e}\right) \boldsymbol{\eta}^{e} \\
\boldsymbol{\beta}^{E}\left(R_{T}^{i}\right) \boldsymbol{\eta}^{i}
\end{array}\right] \boldsymbol{A}=\left[\begin{array}{c}
\boldsymbol{\beta}^{E}\left(R_{T}^{e}\right) \boldsymbol{c}_{1} \\
\boldsymbol{c}_{0}
\end{array}\right],
$$

which corresponds to a Tikhonov regularization term in the original normal equation (24). Indeed, the squared-norm of the residual error between the left- and the right-hand side terms in (30) reads as

$$
\left\|\boldsymbol{\beta}^{E}\left(R_{T}^{e}\right) \boldsymbol{\eta}^{e} \boldsymbol{A}-\boldsymbol{\beta}^{E}\left(R_{T}^{e}\right) \boldsymbol{c}_{1}\right\|^{2}+\left\|\boldsymbol{\beta}^{E}\left(R_{T}^{i}\right) \boldsymbol{\eta}^{i} \boldsymbol{A}\right\|^{2}
$$

whence a Tikhonov matrix $\boldsymbol{\Gamma}=\boldsymbol{\beta}^{E}\left(R_{T}^{i}\right) \boldsymbol{\eta}^{i}$, i.e., $\boldsymbol{\Gamma} \in \mathbb{C}^{2 M+1, L}$. The definition of $\boldsymbol{\Gamma}$ implies that the regularization is not operating in a isotropic way along the $2 M+1$ modal dimensions, and is rather controlled by the modal coefficients expected for the radiation over the internal test surface $\Sigma_{T}^{i}$.

\section{Approximate solutions}

As opposed to the above procedure, alternative approximate approaches can be defined for the computation of layer weights. Two such solutions are here considered, one obtained from the asymptotic development of Hankel's functions, while the second is based on a local reasoning looking at current layers as a collection of directive sources.

The simplicity of these approximate solutions makes them attractive alternatives to the optimal solution presented in the previous section. A direct comparison of their respective accuracy is presented in sec. 8 .

\subsection{Asymptotic solution}

Starting from the asymptotic expressions of $\eta_{m}^{i, e}(R)$ in (29), the problem defined by setting the constraints $\eta_{m}^{i}=0$ and $\eta_{m}^{e}=$ 1 can be solved in closed form. For the special case of $L=2$, the result is given by

$$
\begin{aligned}
& A_{1}=-A_{2} \mathrm{e}^{-\mathrm{j} 2 k_{o} d} \\
& A_{2}=\frac{1}{1-\mathrm{e}^{-\mathrm{j} 2 k_{0} d}} .
\end{aligned}
$$

Since (32) were obtained by requiring minimal modal distortions, the above procedure does not aim at reproducing the field distribution on a global scale as (25), but rather attempts to optimize the modal weights on an individual basis. As shown in sec. 8, while this procedure provides results similar to those obtained with the optimal procedure over the inner region $\Omega_{i}^{\prime}$, its performance in the outer region is definitely worse.

\subsection{End-fire approach}

An alternative approximate solution can be proposed by looking at Love's formulation from a different viewpoint. Consider the electric and magnetic equivalent currents it defines at a generic position $\boldsymbol{r} \in \Sigma$; these two elementary sources make up the smallest directive source, sometimes referred to as a Huygens' source. Inward radiation along $-\hat{\boldsymbol{r}}$ can be demonstrated to be nil.

This observation provides a clue as to how multiple layers of electric currents can emulate the results of the equivalence theorem, by forcing the radiation from a second layer to interfere destructively with the first one along inward directions, while summing up in phase for outward directions. Such a configuration can be interpreted as a (continuous) collection of end-fire arrays, oriented radius-wise; within this framework, the layer weights $A_{1}$ and $A_{2}$ are

$$
\begin{aligned}
& A_{1}=-A_{2} \mathrm{e}^{-\mathrm{j} k_{o} d} \\
& A_{2}=\frac{1}{1-\mathrm{e}^{-\mathrm{j} 2 k_{o} d}},
\end{aligned}
$$



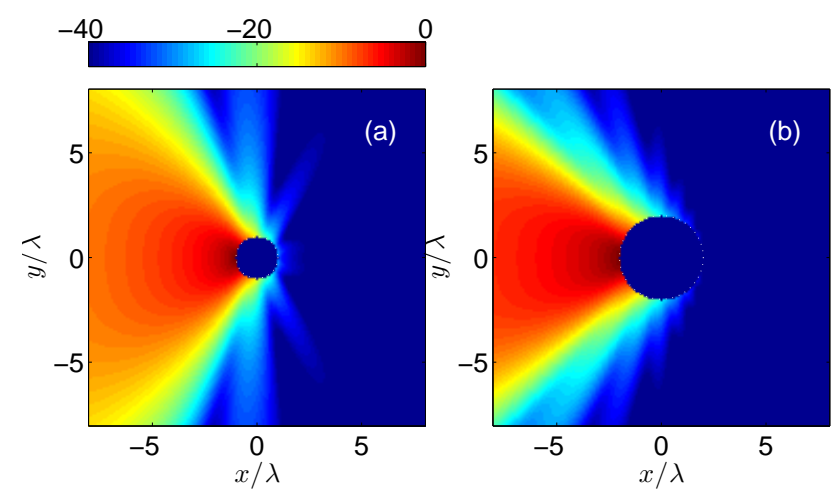

Figure 4: Longitudinal electric-field distribution generated by original sources with: (a) $W_{s}=2 \lambda$ and (b) $W_{s}=4 \lambda$. The inner regions with null radiation marks the minimum-sized cylinders containing the original sources.

which are similar to those found in (32). The main difference, apart the way the results were derived, is that in the case of the end-fire approach the excitation of the two radiating elements is the same apart for a phase shift, as usually done for this kind of arrays, whereas in the asymptotic case we still compute two different auxiliary current distributions. The possibility of generating the information for the second layer from only a single one can be an asset when these data are measured rather than computed, e.g., from near-field facilities, as it would cut the measurement time by half.

The end-fire approach falls short of the global approach employed in the optimal procedure as it does not aim to control the modal distortions. As shown in sec. 8, the residual error increases not only in the external region, but also in the internal one, by giving raise to a spurious focusing roughly covering the space occupied by the original source. Still, its overall ability in controlling the modal distortions is not much worse than that displayed by the asymptotic approximation.

\section{Numerical results}

The accuracy and effectiveness of the proposed approaches are assessed for the case of directive sources described by a linear current distribution $\boldsymbol{J}_{s}(\boldsymbol{r})$ of length $W_{s}$

$$
\boldsymbol{J}_{s}(\boldsymbol{r})=\hat{z} \mathrm{e}^{-\mathrm{j} \beta x} \sin \left(\pi x / W_{s}\right) \delta(y) \delta(z) \quad|x| \leq W_{s} / 2,
$$

tapered toward its ends by a sinusoidal profile in order to produce a dominant main lobe. Fig. 4 shows the electric field distribution they produce for $W_{s}=2 \lambda$ and $W_{s}=4 \lambda$. The amplitudes of their modal coefficients are shown in Fig. 5. These configurations will be used throughout this section as references in evaluating the performance of layer coefficients derived by means of the optimal procedure (25), the asymptotic approximation (32) or the end-fire approximation (33).

All of the following results related to the optimal procedure involve the $\left\{\beta_{m}^{E}\right\}$ as LS weights. Differences observed when using the $\left\{\beta_{m}^{H}\right\}$ are discussed in 8.4.

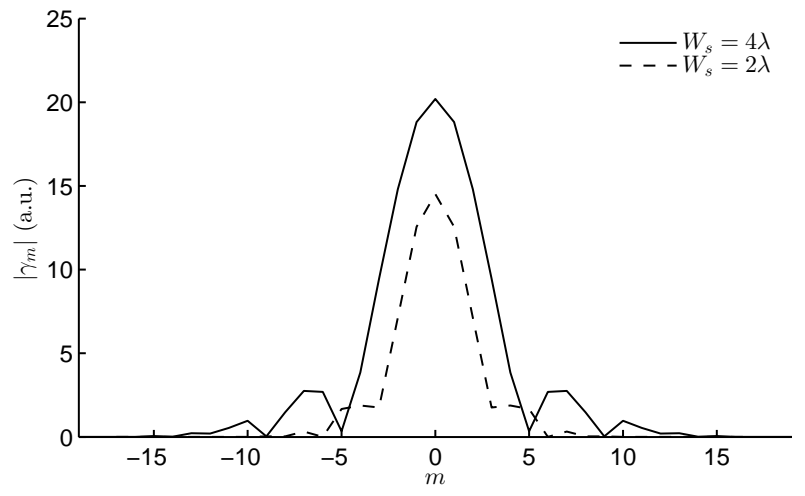

Figure 5: Moduli of the modal coefficients $\left\{\gamma_{m}\right\}$ for the case of $W_{s}=2 \lambda$ and $W_{s}=4 \lambda$, serving as references.

\subsection{Modal distortions}

The question of how accurately two layers of electric currents can emulate these configurations can be assessed by means of several indicators. The first here considered, perhaps the most straightforward, is the set of modal distortions $\left\{\eta_{m}\right\}$ for the external and internal modal coefficients (16), which account for the imperfect reproduction of the original modal coefficients.

Some examples of results for the external distortions are given in Fig. 6, for three values of the inner radius $R_{1}$; the case $R_{1}=W_{s} / 2$ corresponds to the boundary of the minimum cylinder containing the original source. Unless otherwise mentioned in the rest of the paper, the second layer is always found at a distance $d=\lambda / 4$ outside the first one.

Fig. 6 shows that the end-fire approximation, though yielding a limited error over the modal indexes $m$ where most of the radiated energy is found (see Fig. 5), actually presents a rapidly diverging error for $|m|>10$. More fundamentally, $\eta_{m}^{e}$ for the end-fire case present a systematic error (bias) that will be shown in sec. 8.3 to have a major impact on the field topographies.

On the contrary, the optimal procedure has a very good performance for low-order modal indexes, where the $\left\{\beta_{m}\right\}$ coefficients enforce the most stringent conditions for the LS algorithm. It can be noticed how this performance comes to the expenses of a distortion that peaks for $|m|=12$, though the distortions are always bounded in these examples and have a minor impact on the overall results, since they apply to modes that are very weakly excited.

Increasing $R_{1}$ external distortions flatten out over a wider range of modes for the three approaches, but the end-fire approximation still displays a flat residual offset, as shown in Fig. 6(c). The differences in the performances of the three solutions are highlighted in Fig. 7, where $\left|\eta_{m}^{e}-1\right|$ is shown to be only minimized in the case of optimal layer weights, while the asymptotic solution is only slightly better than the end-fire one.

Concerning the internal distortions, Fig. 8 confirms that the results obtained with the optimal and asymptotic solutions are very similar, while the end-fire solution is again characterized by a flat offset that explains the appearance of a relatively important inner radiation, as shown in sec. 8.3. Again, setting auxiliary currents further away from the original source region 

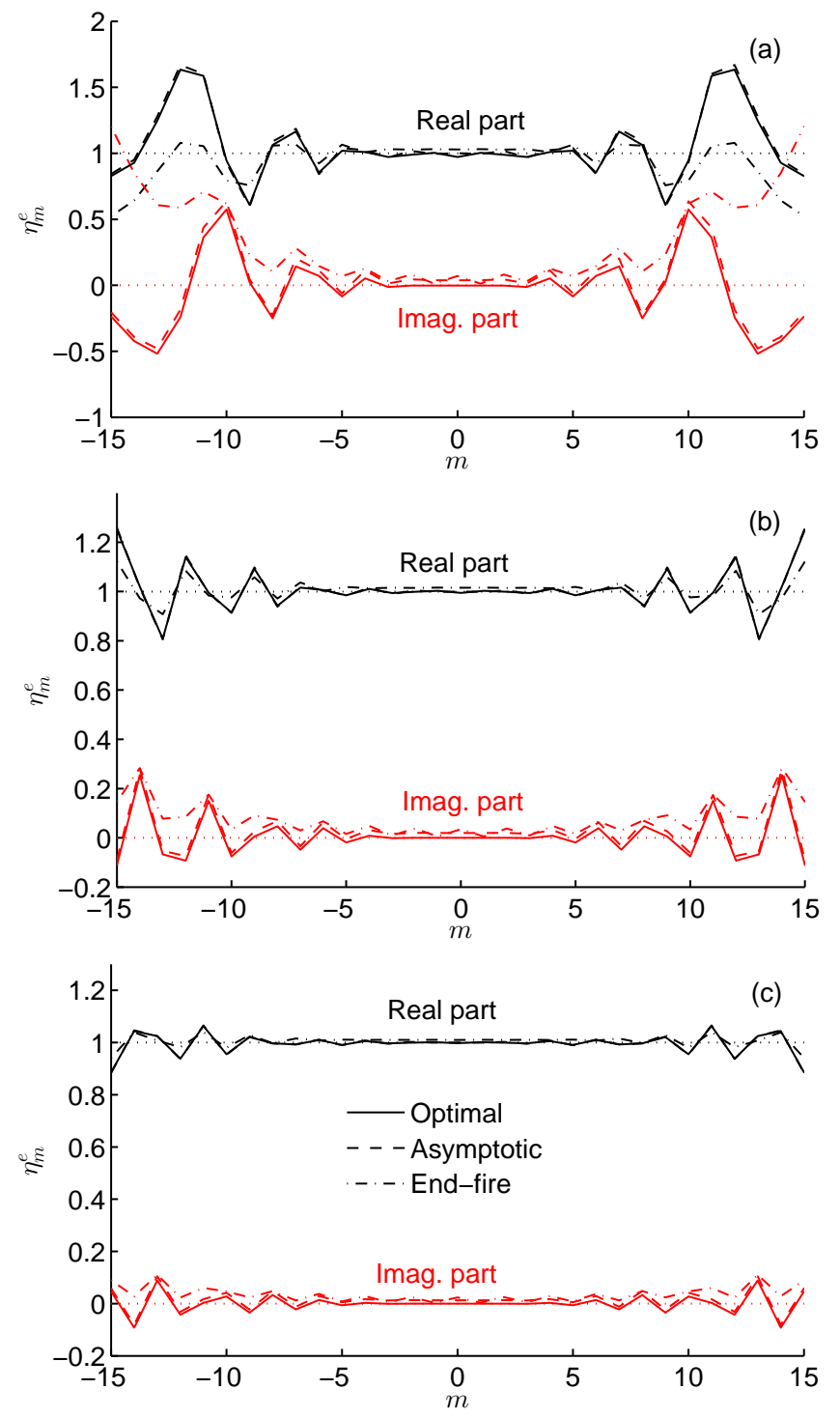

Figure 6: Real and imaginary parts of the overall external-field distortions $\left\{\eta_{m}^{e}\right\}$, computed in the case $W_{s}=4 \lambda$ and $\Delta_{R}=\lambda / 4$ for: (a) $R_{1} / W_{s}=1 / 2$; (b) $R_{1} / W_{s}=$ 1 and (c) $R_{1} / W_{s}=3 / 2$.

strongly reduces the absolute value of the internal distortions; at the same time, the internal distortions also increase at a lower rate. It is worth observing that this trend is not shared by the end-fire solution, as its performance over low-order modes remains virtually unchanged.

\subsection{Field errors on test surfaces}

The modal distortions translate into errors on the electromagnetic field generated by the auxiliary sources, which can be measured on test surfaces as discussed in sec. 4. An example of typical results is given in Figs. 9 and 10, for the case $R_{1} / W_{s}=1 / 2$ for end-fire and optimal procedures. In these two cases, internal (external) test surfaces were defined at half a wavelength inside (outside) the innermost (outermost) layer of auxiliary electric currents. These results show that although the optimal procedure is expected to ensure a better performance,

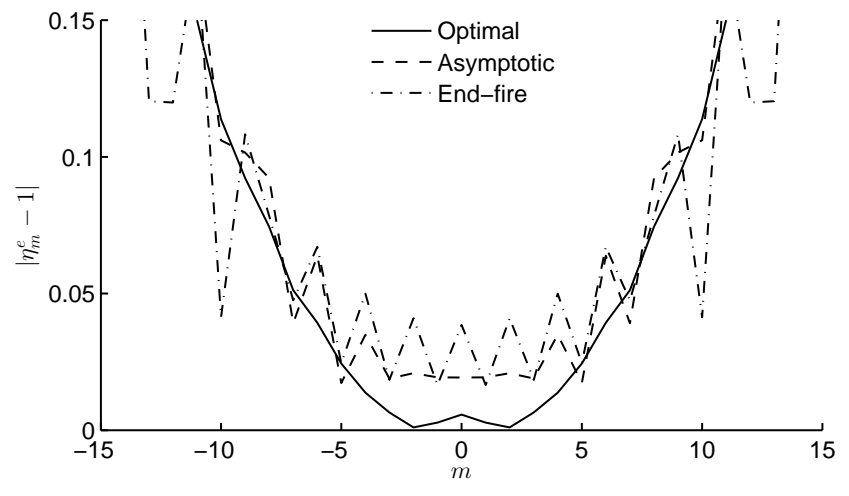

Figure 7: The distance $\left|\eta_{m}^{e}-1\right|$, computed in the case $W_{s}=4 \lambda$ and $\Delta_{R}=\lambda / 4$ for $R_{1} / W_{s}=1$.

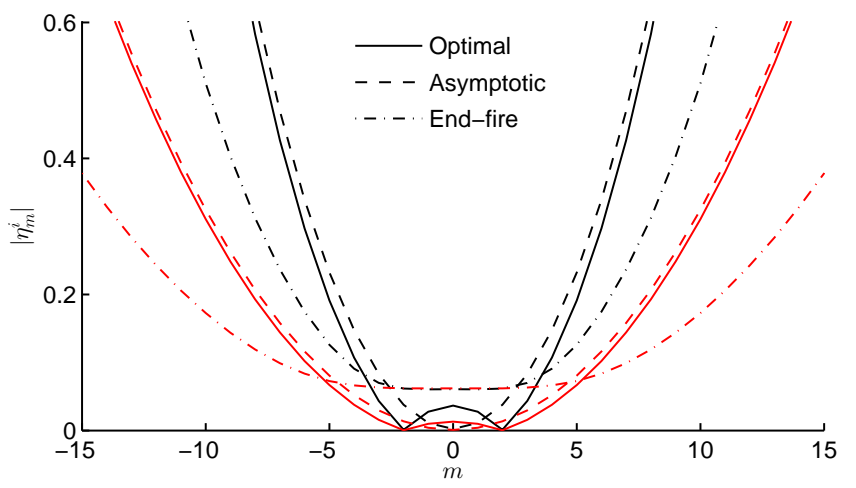

Figure 8: Absolute value of the overall internal-field biases $\left\{\eta_{m}^{i}\right\}$, computed in the case $W_{s}=4 \lambda$ with $\Delta_{R}=\lambda / 4$, for $R_{1} / W_{s}=1 / 2$ (black lines) and $R_{1} / W_{s}=$ $3 / 2$ (red lines).

the residual errors over the internal test surface can be locally stronger than by applying the end-fire approach. Moreover, the optimal procedure ensures that these errors are on average equal to zero, as opposed to the end-fire approximation; as it will be shown in sec. 8.3, these coherent deviations lead to residual focusing over the inner region.

Residual errors can be summarized by defining relative errors for the electric and magnetic fields

$$
\epsilon_{E, H}^{2}\left(R_{T}\right)=\frac{e_{E, H}^{2}\left(R_{T}\right)}{\mathscr{E}_{E, H}},
$$

where $\mathscr{E}_{E, H}$ stands for the square of the $L_{2}$ norm of the electric (magnetic) field over the external test surface $\Sigma_{T}^{e}$. Recalling (15), (17) and (21)

$$
\begin{aligned}
\epsilon_{E, H}^{2}\left(R_{T}^{e}\right) & =\frac{\sum_{m=-\infty}^{\infty}\left|\eta_{m}^{e}-1\right|^{2}\left|\beta_{m}^{E, H}\left(R_{T}^{e}\right)\right|^{2}}{\sum_{m=-\infty}^{\infty}\left|\beta_{m}^{E, H}\left(R_{T}^{e}\right)\right|^{2}} \\
\epsilon_{E, H}^{2}\left(R_{T}^{i}\right) & =\frac{\sum_{m=-\infty}^{\infty}\left|\eta_{m}^{i}\right|^{2}\left|\beta_{m}^{E, H}\left(R_{T}^{i}\right)\right|^{2}}{\sum_{m=-\infty}^{\infty}\left|\beta_{m}^{E, H}\left(R_{T}^{e}\right)\right|^{2}},
\end{aligned}
$$



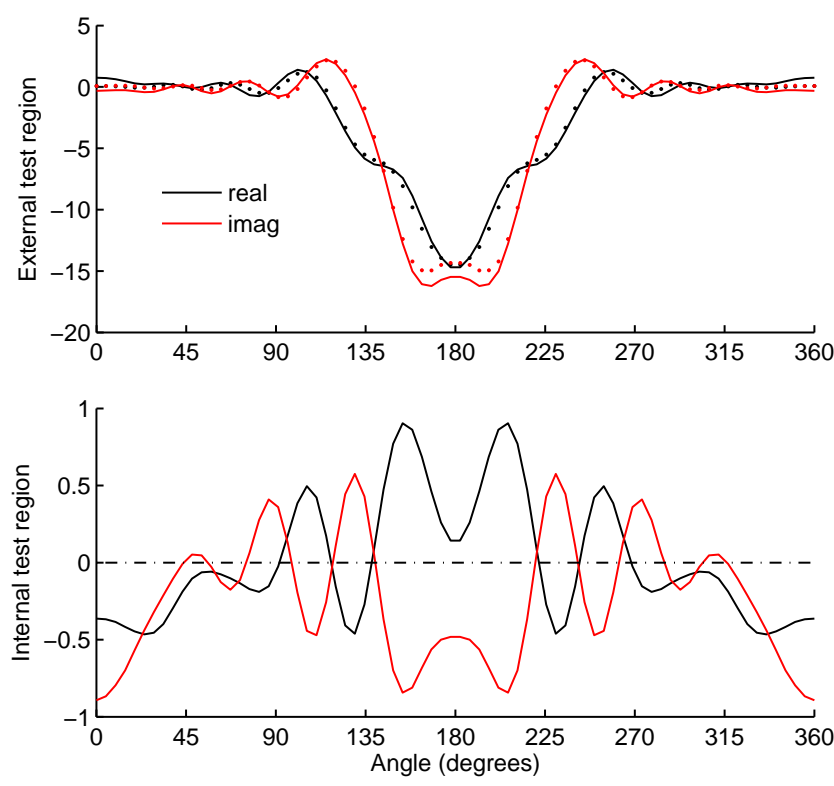

Figure 9: Real and imaginary parts of the longitudinal electric field for $W_{s}=4 \lambda$, $R_{1} / W_{s}=1 / 2$ and $\Delta R=\lambda / 4$, computed over cylindrical external (top figure) and internal (bottom figure) test surfaces, with $R_{T}^{i}=R_{1}-\lambda / 2$ and $R_{T}^{e}=R_{2}+\lambda / 2$, respectively. Results obtained under the end-fire approximation are shown as solid lines, reference results as dots.

where $\mathscr{E}_{E, H}$ are also taken as references for the internal errors, in order to assess the relative intensity of the residual field generated over the inner region $\Omega_{i}^{\prime}$.

These figures of merit are shown in Figs. 11 to 13. Several trends can be pointed out. First of all, as $\Delta R$ approaches $\lambda / 2$, residual errors increase. This result can be understood by noticing that, under an asymptotic approximation, the field distributions observed over two layers associated to each mode will be proportional one to the other if they are half a wavelength apart. Hence, rather than conveying complementary information that could be made to interfere, the two sets of auxiliary currents become increasingly redundant.

At the same time, as the ratio $R_{1} / W_{s}$ increases, the errors systematically reduce. This behavior is easily understood within the framework introduced in sec. 6: in the limit for $k_{0} R_{1} \gg 1$, all modal coefficients can be controlled at the same time. Fundamentally for the same reason, errors on the magnetic and electric fields over the external test surface are practically identical, while this is not true over the internal test surface, where the asymptotic expressions do not hold.

As expected, all errors are consistently lower for the optimal procedure. More importantly, the optimal procedure takes better advantage of a larger $R_{1} / W_{s}$, with a faster rate of reduction in the errors, when compared with the two approximate cases. The performance of the asymptotic approximation approaches that of the optimal procedure for larger $R_{1} / W_{s}$, since the former is correct in the limit for $k_{0} R_{1} \rightarrow \infty$.
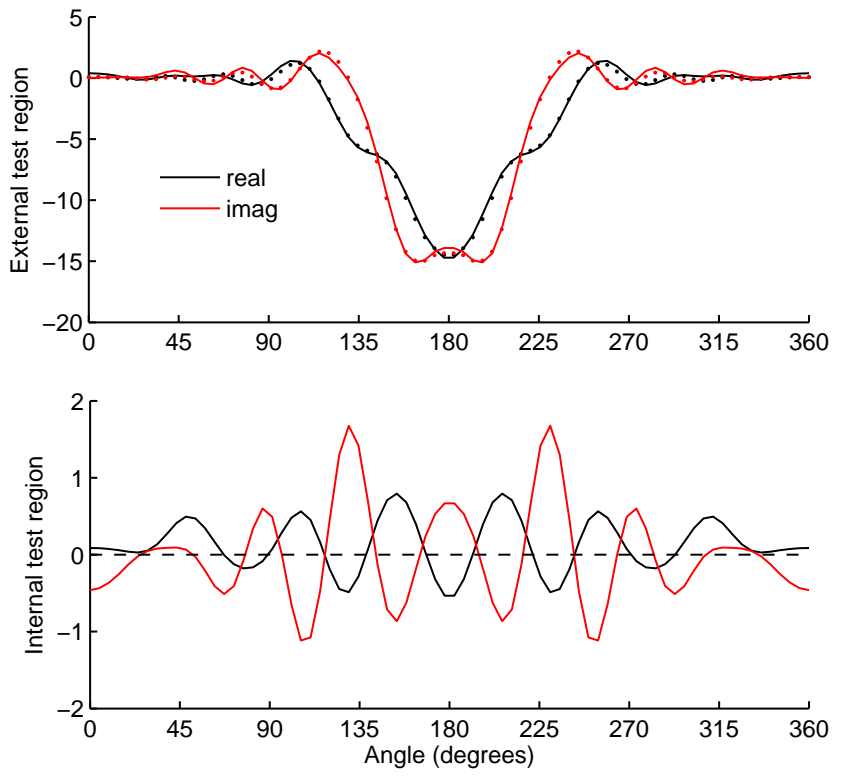

Figure 10: Same configuration as in Fig. 9. Results obtained with optimal layer weights.

\subsection{Field topographies}

An example of the field topographies generated by two-layer auxiliary currents is shown in Fig. 14, emulating the linear source (34) when $W_{s}=4 \lambda$. The three sets of electric and magnetic field distributions are to be compared to the original case reproduced in Fig. 4(b).

Differences between the performance of the three solutions are shown in Figs. 15 and 16, as percentages of the peak field amplitude observed over the external region $\Omega_{e}^{\prime}$; two values of $R_{1} / W_{s}$ are considered, namely 0.5 and 1.5 . Independently from the dimensions of the shell-region, it is found that the end-fire approach always leads to a residual (coherent) focusing within the inner region $\Omega_{i}^{\prime}$, as opposed to an incoherent interference pattern observed with the other two solutions. Errors in the external region drop when applying the optimal procedure.

\subsection{Cross comparisons}

This section draws some cross comparisons between the performance as already presented and the layer weights that generated them. The first such comparison is presented in Table 1 where the layer weights applied for the three methods are summarized. The weights $\left\{A_{l}\right\}$ are presented in a normalized form, as $A_{2} / A_{1}$.

We first need to recall that the weights of the approximate solutions only depend on the relative distance between the layers. By the same token, the approximate solutions are by definition only operating by phase-shifting the individual radiations from the auxiliary layers. Conversely, for the optimal procedure, this property is never explicitly enforced; the fact that all the results in Table 1 involve $\left|A_{1} / A_{2}\right|=1$ is therefore worth noticing, as it implies that there is no need for amplitude-compensation schemes. 

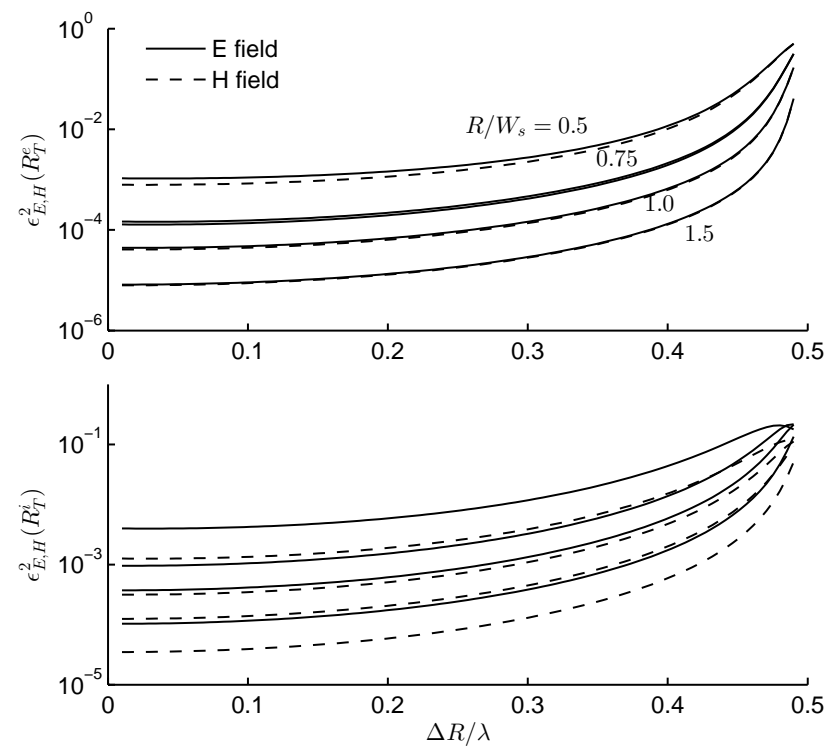

Figure 11: Comparison between relative errors on the electric and magnetic fields over the external test surface $\Sigma_{T}^{e}$ (upper graph), with $R_{T}^{e}=R+\Delta R+\lambda / 2$ and the internal test surface $\Sigma_{T}^{i}$ (lower graph), with $R_{T}^{i}=R-\lambda / 2$, for a varying distance $\Delta R$ between two current layers. Four values of $R / W_{s}$ are considered, equal to $0.5,0.75,1.0$ and 1.5 . Results for a source width $W_{s}=4 \lambda$, optimal layer weights.

As a consequence, it appears that the major differences in the performance between the approximate and optimal procedures are in fact due to minor differences in the phase-shift angle enforced between the layers. While the asymptotic solution requires an in-phase radiation of the two layers, the optimal one spans phase-shift angles between 0.26 and 2.2 degrees, for $R_{1} / W_{s}$ passing from 1.5 to 0.5 . Correspondingly, the external error decreases more steeply for the optimal procedure, with a relative improvement of about a factor 2 to 10 with respect to the asymptotic solution.

Table 2 seeks to point out how the choice of electric or magnetic weights $\left\{\beta_{m}\right\}$ affects the performance of the optimal procedure. To this end, the layer weights and errors were computed for the two choices of $\left\{\beta_{m}\right\}$ : all the quantities in Table 2 noted by the letter ' $R$ ' stand for the ratio of results obtained by choosing $\left\{\beta_{m}^{H}\right\}$ and those obtained with $\left\{\beta_{m}^{E}\right\}$; e.g., $R_{A}$ is the ratio of $A_{2} / A_{1}$ obtained in the two cases, of which only the marginal phase-shift angle is shown, as expressed in degrees. All other ratios are self-explaining.

It can be concluded that the kind of $\left\{\beta_{m}\right\}$ chosen has little impact on the error metrics on the external test surface, with variations of the order of the percent point; this trend is reinforced when passing from $W_{s}=2 \lambda$ to $W_{s}=4 \lambda$. Moreover, the same conclusions hold for both electric and magnetic field external errors. As opposed to these conclusions, the internal errors are more sensitive to the choice of the $\left\{\beta_{m}\right\}$ weights.

The asymptotic proportionality between $\left\{\beta_{m}^{E}\right\}$ and $\left\{\beta_{m}^{H}\right\}$, discussed in sec. 5 , is confirmed, with residual phase-shift angle of $R_{A}$ passing from 1.8 to 0.23 degrees for $W_{s}=2 \lambda$.

A last comparison is needed between the performance of the
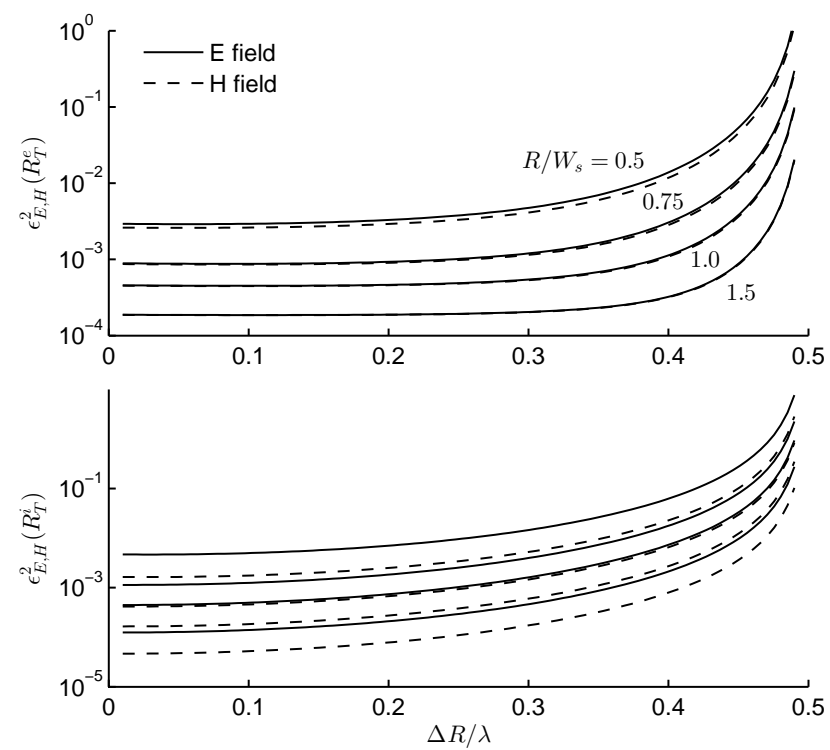

Figure 12: Same configuration as in Fig. 11; results for the asymptotic solution.

\begin{tabular}{cccccc}
\hline \hline$R_{1} / W_{s}$ & Type & $\left|A_{2} / A_{1}\right|$ & $\angle A_{2} / A_{1}$ & $\epsilon_{E}^{2}\left(R_{T}^{e}\right)$ & $\epsilon_{E}^{2}\left(R_{T}^{i}\right)$ \\
\hline \multirow{3}{*}{0.5} & ef & 1.0 & 90 & $5.910^{-3}$ & $5.610^{-3}$ \\
& as & 1.0 & 0.0 & $3.810^{-3}$ & $9.510^{-3}$ \\
& opt & 1.0 & 2.2 & $1.910^{-3}$ & $7.910^{-3}$ \\
\hline \multirow{3}{*}{1.0} & ef & 1.0 & 90 & $1.010^{-3}$ & $1.810^{-3}$ \\
& as & 1.0 & 0.0 & $4.910^{-4}$ & $1.010^{-3}$ \\
& opt & 1.0 & 0.59 & $9.310^{-5}$ & $8.510^{-4}$ \\
\hline \multirow{3}{*}{1.5} & ef & 1.0 & 90 & $4.310^{-4}$ & $1.010^{-3}$ \\
& as & 1.0 & 0.0 & $1.910^{-4}$ & $2.910^{-4}$ \\
& opt & 1.0 & 0.26 & $1.810^{-5}$ & $2.410^{-4}$ \\
\hline \hline
\end{tabular}

Table 1: Absolute value and phase shift of the relative weight $A_{2} / A_{1}$, for the three methods of computation of the layer weights, for the case $W_{s}=4 \lambda$ and $\Delta R=\lambda / 4$. The corresponding relative errors $\epsilon_{E}^{2}\left(R_{T}\right)$ give a measure of how such small adjustments in the layer weights lead to large differences in the quality of reconstruction of the field distribution. Shorthand notations are used for the end-fire (ef), asymptotic (as) and optimal (opt) solutions.

external-field optimization and the joint internal-external one. It was suggested in sec. 6 that, asymptotically, reproducing the original radiation over the external region must pass for a null radiation over the internal region. As such, a joint optimization can be regarded as redundant, as long as $L \ll M$. In this respect, the results shown in Fig. 17 support this analysis, since the internal modal distortions are practically unmodified when switching between the two optimization strategies. The joint optimization should therefore be considered only in those cases where $L \simeq M$.

\section{Conclusions}

This paper analyzed the possibility of defining auxiliary current distributions, extending the idea of Love's equivalent theorem to the case of electric-only currents. Rather than proceeding through the definition of an inverse problem, our proposal is 

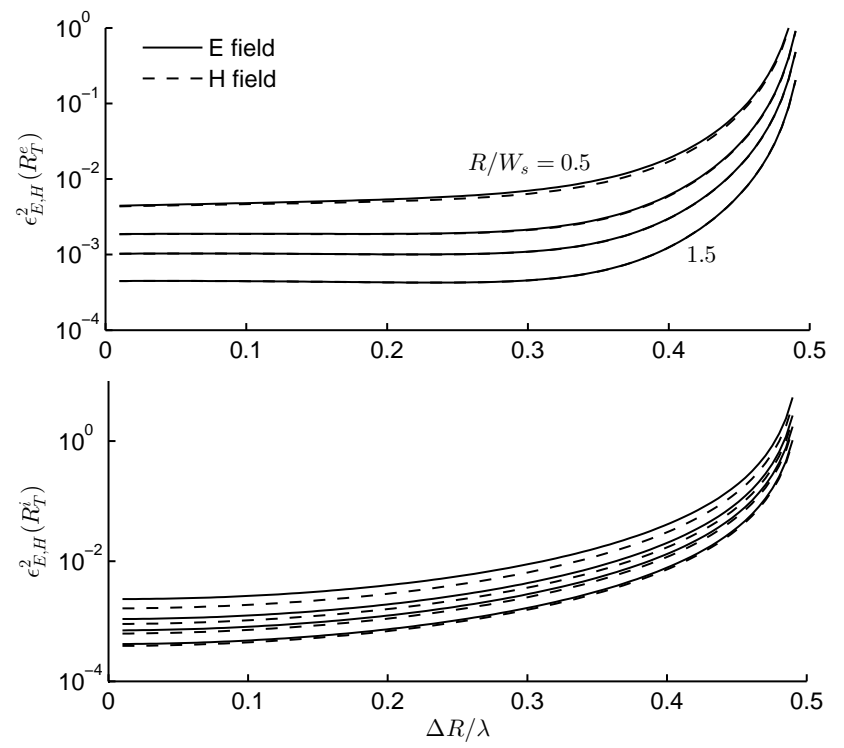

Figure 13: Same configuration as in Fig. 11; results for the end-fire approximation.

\begin{tabular}{ccccccc}
\hline \hline$R_{1} / W_{s}$ & $W_{s} / \lambda$ & $\angle R_{A}$ & $R_{\epsilon, e}^{E}$ & $R_{\epsilon, i}^{E}$ & $R_{\epsilon, e}^{H}$ & $R_{\epsilon, i}^{H}$ \\
\hline \multirow{2}{*}{0.5} & 2 & 1.79 & 1.03 & 1.25 & 1.01 & 0.543 \\
& 4 & 0.447 & 0.969 & 1.02 & 0.951 & 0.987 \\
\hline \multirow{2}{*}{1.0} & 2 & 0.499 & 1.05 & 1.25 & 1.04 & 0.511 \\
& 4 & 0.119 & 1.01 & 0.991 & 1.01 & 0.975 \\
\hline \multirow{2}{*}{1.5} & 2 & 0.234 & 1.05 & 1.26 & 1.05 & 0.505 \\
& 4 & 0.0553 & 0.994 & 1.01 & 0.993 & 0.973 \\
\hline \hline
\end{tabular}

Table 2: Comparison of the performance of the optimal procedure when applying magnetic or electric weights $\left\{\beta_{m}\right\}$. All quantities represent the ratio of results and metrics obtained with the magnetic weights normalized to those obtained with electric weights. Refer to sec. 8.4 for details about the notations.

based on a hybrid solution, keeping the electric current distribution defined by Love's theorem. The role of magnetic currents is taken over by further layers of electric currents weighted in such a way as to approximate the results expected for the equivalence theorem. The resulting procedure ensures a good performance, with residual errors of the order of a few percent points.

\section{Acknowledgement}

Part of the work here reported was funded by the French National Research Agency through the grant ANR-12-ASTR0005, within the framework of the 2012 ASTRID program.

\section{References}

[1] A. Love, The integration of the equations of propagation of electric waves., Proceedings of the Royal Society of London 68 (442-450) (1901) 19-21.

[2] H. MacDonald, The integration of the equations of propagation of electric waves, Proceedings of the London Mathematical Society 2 (1) (1912) 9195.
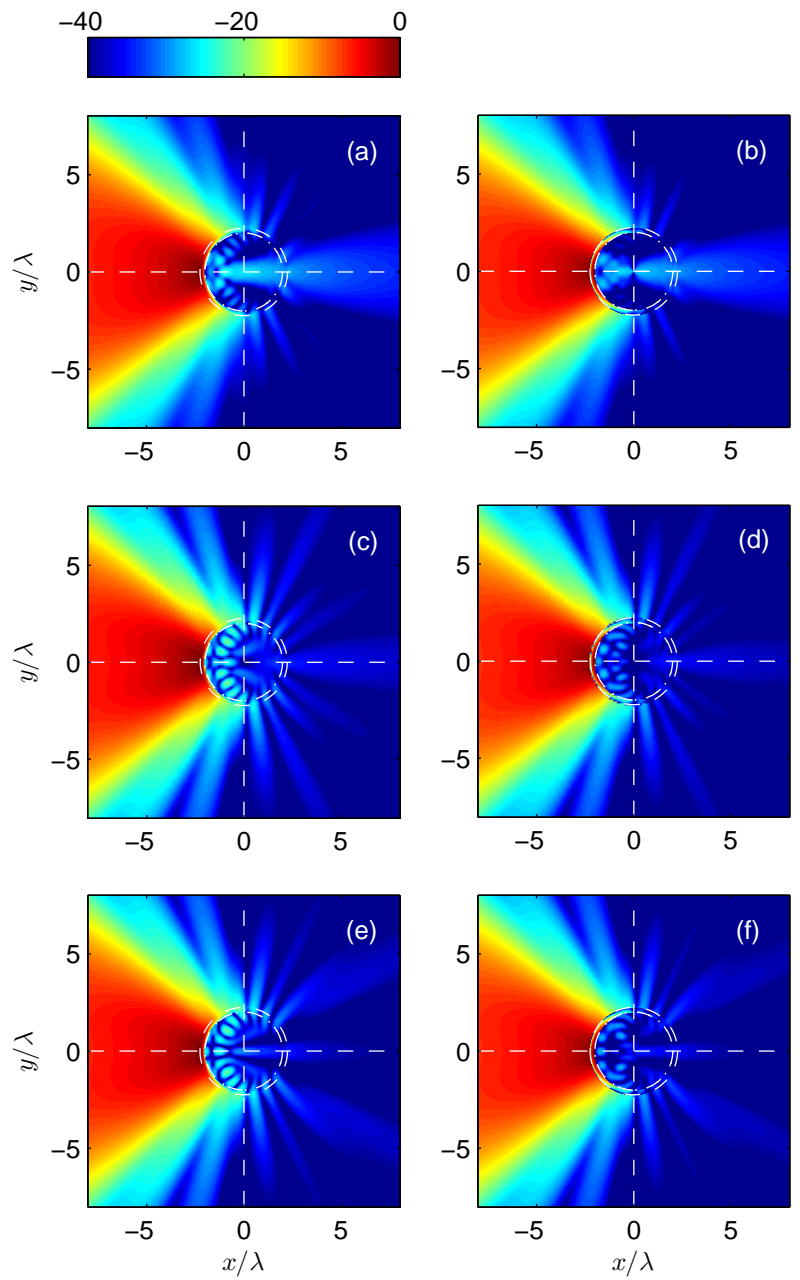

Figure 14: Field distributions generated by two-layer auxiliary currents with $R_{1}=W_{s} / 2$ and spacing $\Delta R=\lambda / 4$, for the source (34) with $W_{s}=4 \lambda$. The modulus of the longitudinal electric (left column) and transverse magnetic (right column) fields, expressed in $\mathrm{dB}$ and normalized to their peak value, for the (a)-(b) end-fire, (c)-(d) asymptotic and (e)-(f) optimal solutions. The auxiliarycurrent layers are shown as dashed circles.

[3] W. Smythe, The double current sheet in diffraction, Physical Review 72 (11) (1947) 1066.

[4] R. Harrington, Time-Harmonic Electromagnetic Fields, McGraw-Hill, New York, NY, 1961.

[5] S. R. Rengarajan, Y. Rahmat-Samii, The field equivalence principle: Illustration of the establishment of the non-intuitive null fields, Antennas and Propagation Magazine, IEEE 42 (4) (2000) 122-128.

[6] S. Schelkunoff, Some equivalence theorems of electromagnetics and their application to radiation problems, Bell Syst. Tech. J 15 (1) (1936) 92.

[7] E. Martini, G. Carli, S. Maci, An equivalence theorem based on the use of electric currents radiating in free space, Antennas and Wireless Propagation Letters, IEEE 7 (2008) 421-424.

[8] F. Las-Heras, T. K. Sarkar, Radial field retrieval in spherical scanning for current reconstruction and nf-ff transformation, Antennas and Propagation, IEEE Transactions on 50 (6) (2002) 866-874.

[9] J. Colinas, Y. Goussard, J. Laurin, Application of the Tikhonov regularization technique to the equivalent magnetic currents near-field technique, Antennas and Propagation, IEEE Transactions on 52 (11) (2004) 31223132.

[10] P.-A. Barriere, J. Laurin, Y. Goussard, Mapping of equivalent currents on high-speed digital printed circuit boards based on near-field measurements, Electromagnetic Compatibility, IEEE Transactions on 51 (3) 

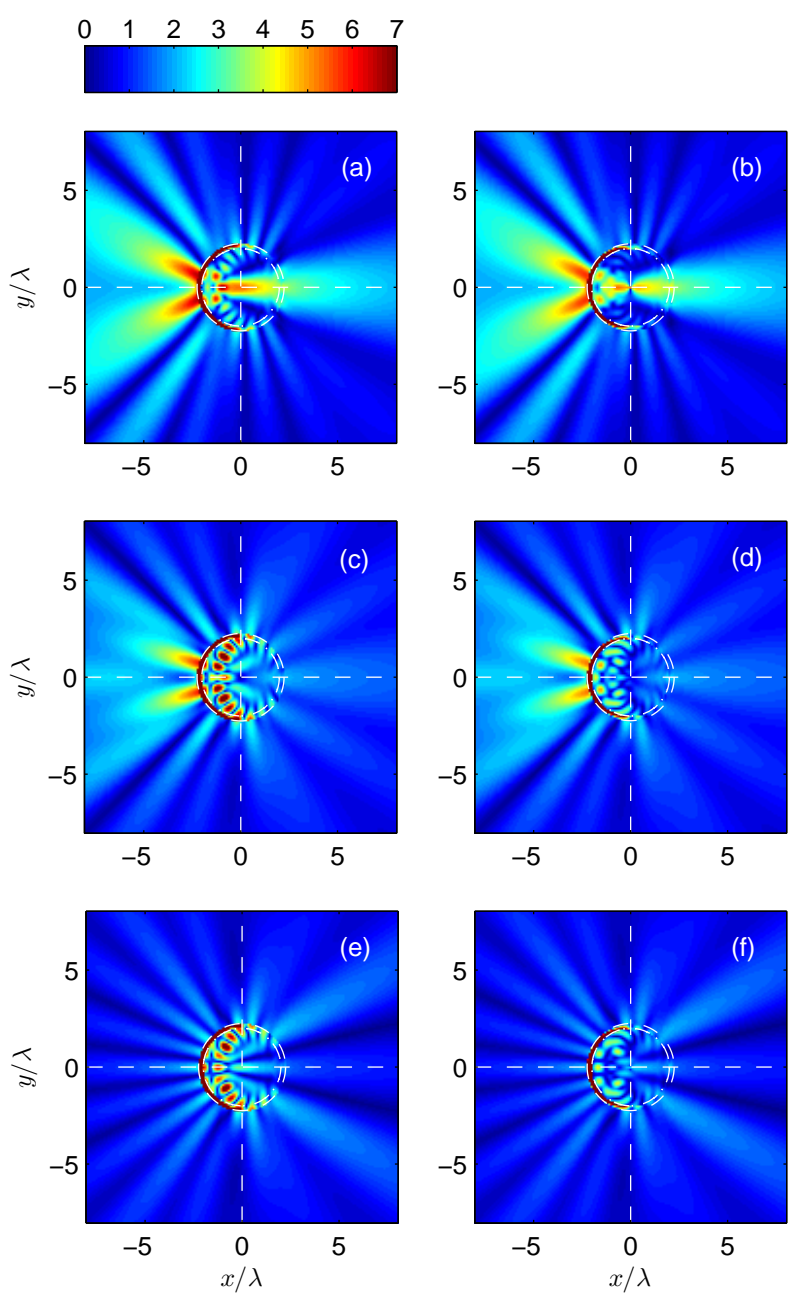

Figure 15: Intercomparison between the errors of the three synthesis methods, expressed as percentage of the peak amplitude of the field distributions external to the auxiliary-current layers, for $R_{1} / W_{s}=1 / 2$ (cf. Fig. 14). Axial electric (left column) and transverse magnetic (right column) error distributions for the (a)-(b) end-fire, (c)-(d) asymptotic and (e)-(f) optimal solutions.

(2009) 649-658.

[11] T. F. Eibert, E. Kaliyaperumal, C. H. Schmidt, et al., Inverse equivalent surface current method with hierarchical higher order basis functions, full probe correction and multilevel fast multipole acceleration, Progress In Electromagnetics Research 106 (2010) 377-394.

[12] J. Van Bladel, Electromagnetic fields, IEEE, 2007.

[13] J. E. Hansen, Spherical near-field antenna measurements, Vol. 26, IET, 1988.

[14] W. C. Chew, E. Michielssen, J. Song, J. Jin, Fast and efficient algorithms in computational electromagnetics, Artech House, Inc., 2001.

[15] C. L. Lawson, R. J. Hanson, Solving least squares problems, Vol. 161, SIAM, 1974.
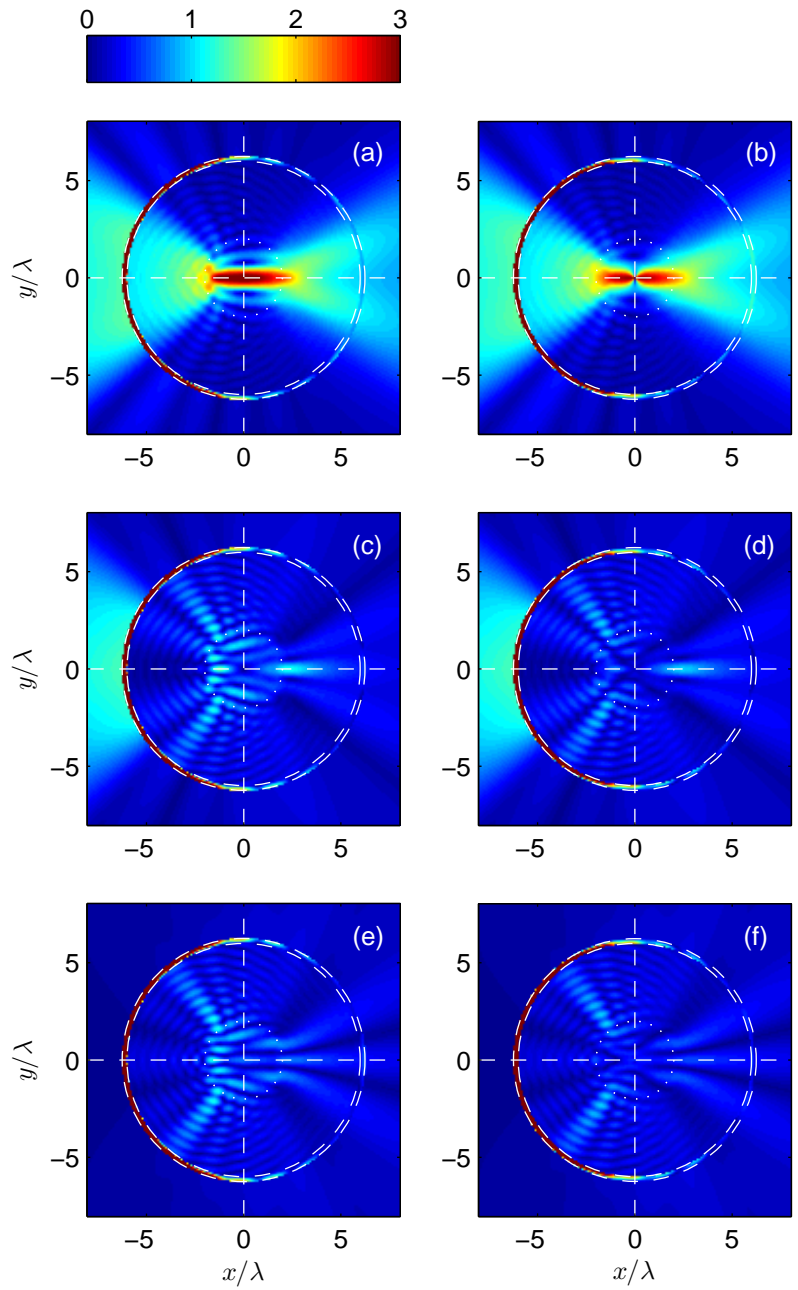

Figure 16: Same as in Fig. 15, for the case $R_{1} / W_{s}=3 / 2$.

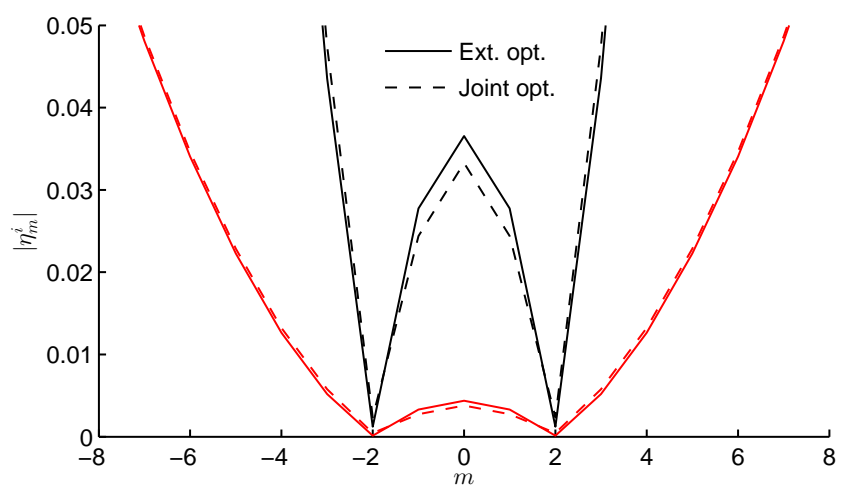

Figure 17: Absolute value of the overall internal-field modal distortions $\left\{\eta_{m}^{i}\right\}$, computed in the case $W_{s}=4 \lambda$ with $\Delta_{R}=\lambda / 4$, for $R_{1} / W_{s}=1 / 2$ (black lines) and $R_{1} / W_{s}=3 / 2$ (red lines). 